\title{
Identificación de polímeros por espectroscopía infrarroja"
}

Fecha de recepción: 03 de agosto de 2017

Fecha de aprobación: 17 de octubre de 2017
Javier Ricardo Velandia Cabral

Universidad EAN

jrvelandia@universidadean.edu.co

DOI: https://doi.org/10.21158/23823399.v5.n0.2017.2005

Cómo citar este artículo/To reference this article/Comment citer cet article/Para citar este artigo: Velandia Cabra, J. R. (2017). Identificación de polímeros por espectroscopía infrarroja. Revista Ontare. $5, \mathrm{p} 115-140$

\section{Resumen}

Los polímeros son uno de los materiales más utilizados en la actualidad. Dadas sus propiedades, el ser humano los ha convertido en parte de la mayoría de los objetos de la vida diaria. Sin embargo, la vida útil de muchos productos es muy corta en la mayoría de los casos y esto los ha convertido en un problema por la contaminación que generan.

Una de las principales dificultades es la de separar estos materiales de acuerdo al tipo de polímero para facilitar su reciclaje. Es aquí donde la labor de identificar y separar los polímeros es la etapa crítica de este proceso. (Ignatyev, Thielemans, y Vander Beke, 2014)

El objetivo de este artículo es el de introducir brevemente la técnica de Espectroscopía infrarroja, el funcionamiento de un espectrómetro infrarrojo y mostrar algunos resultados de análisis realizados en algunos polímeros comerciales.

\section{Palabras clave}

Espectroscopía infrarroja; polímeros; monómeros; bandas de absorción infrarroja; reflexión total atenuada; número de onda; reciclaje.

\footnotetext{
Se agradece en especial al profesor Eduardo Ramírez Valencia, director de los laboratorios de la Facultad de Ingeniería, por permitir realizar este estudio y a la Universidad EAN por hacer posible el uso del laboratorio y del equipo.

1 Ingeniero químico de la Universidad Nacional de Colombia. ORCID: https://orcid.org/0000-00022650-5547
} 


\title{
Identification of polymers by infrared spectroscopy
}

\begin{abstract}
Polymers are one of the most used materials nowadays. Given their properties, the human being has made of them part of most of the objects of daily life. However, the lifespan of these products is very short in most cases and this has turned them into a problem due to the pollution they generate.

One of the main difficulties is to separate these materials according to the type of polymer in order to facilitate their recycling. This is where the task of identifying and separating polymers becomes the critical stage of this process. (Ignatyev, Thielemans, \& Vander Beke, 2014)

The aim of this article is to briefly introduce the infrared spectroscopy technique and the operation of an infrared spectrometer, and show some results of analyzes performed on some commercial polymers.
\end{abstract}

\section{Keywords}

Infrared spectroscop; polymers; monomers; infrared absorption bands; attenuated total reflection; wave number; recycling.

\section{Identification des polymères par spectroscopie infrarouge}

\section{Résumé}

Les polymères font partie des matériaux les plus utilisés actuellement du fait de leurs propriétés très recherchées et font désormais partie intégrante de la plupart de nos objets quotidiens. La durée de vie de nombre de ces produits est néanmoins très courte les rendant problématiques du fait de la pollution qu 'ils génèrent. L'une des principales difficultés rencontrées reside dans la séparation des matériaux en fonction du type de polymère pour en faciliter leur recyclage. Cette identification et séparation est donc l'étape critique de ce processus. (Ignatyev, Thielemans, \& Vander Beke, 2014). 
L'objectif de cet article est de proposer une brève introduction à la technique de spectroscopie infrarouge, du fonctionnement d'un spectromètre infrarouge et de présenter certains résultats d'analyses effectuées sur différents polymères commerciaux.

\section{Mots clefs}

Pneus usés; broyage mécanique; granulés de caoutchouc recyclé; mélange d'asphalte modifié; contamination environnementale.

\section{Identificação de polímeros por espectroscópia infravermelha}

\section{Resumo}

Os polímeros são um dos materiais mais utilizados na atualidade. Dadas suas propriedades, o ser humano converteu-os em parte da maioria dos objetos da vida diária. No entanto, a vida útil de muitos produtos é muito curta na maioria dos casos e isto os transforma num problema pela contaminação que geram. Uma das principais dificuldades é a de separar estes materiais de acordo com o tipo de polímero para facilitar sua reciclagem. É aqui onde o trabalho de identificar e separar os polímeros é a etapa crítica deste processo (Ignatyev, Thielemans, \& Vander Beke, 2014). O objetivo deste artigo éo de introduzir brevemente a técnica de espectroscópia infravermelha, o funcionamento de um espectrómetro infravermelho e mostrar alguns resultados de análises realizadas em alguns polímeros comerciais.

\section{Palavras-chave}

Espectroscópia infravermelha; polímeros; monómeros; banda de absorção infravermelha; reflexão total atenuada; número de onda; reciclagem. 


\section{Introducción}

Doder separar e identificar los polímeros es una de las principales - dificultades que se encuentran para poder darles uso de nuevo a estos materiales. La espectroscopía infrarroja es una de las técnicas de análisis moderno que permite diferenciar de manera rápida las diferentes clases de polímeros de forma rápida e inequívoca.

Para entender un poco más sobre la problemática de los polímeros y la técnica, se abordarán tres temáticas generales.

\section{Los polímeros y la clasificación para su reciclaje}

Tn polímero se define como grandes moléculas que están compuestas en general de moléculas más pequeñas, subunidades llamadas monómeros que se unen entre sí en un proceso que se conoce como polimerización.

Aunque los polímeros más comunes se basan en uniones covalentes de átomos de carbono, en la actualidad muchos de los polímeros sintéticos lo hacen en uniones de átomos inorgánicos como el silicio (Flory, 1953).

Sin embargo, luego de haberse convertido en productos comerciales y haber terminado su ciclo de vida útil, son materiales desechados. Hasta antes de la década de los noventa, la totalidad de los residuos de polímeros era enterrada o incinerada.

Por la cantidad de desechos de polímeros generados y el problema ambiental que estos representan, se empezaron a realizar esfuerzos para reciclar estos materiales. 
Sin embargo, esta tarea no es fácil. Además de moler y fundir de nuevo el material para convertirlo en un nuevo producto, hay otra serie de inconvenientes técnicos que dificultan esta labor.

A diferencia de otros materiales, como el aluminio de las latas o el vidrio, que se recicla para obtener un material esencialmente idéntico al mismo producto, en los polímeros muy pocas veces se obtienen las mismas propiedades físicas luego del reciclaje (Eisenreich y Rohe, 2006).

La principal dificultad, y la que se considera que tiene mayor influencia en las propiedades físicas de un material polimérico reciclado, es que se haya realizado una adecuada clasificación del material (Escuela Colombiana de Ingeniería, 2008).

Si dos polímeros se mezclan para su reciclaje, se pueden alterar significativamente las propiedades mecánicas del nuevo material y hacerlo menos atractivo comercialmente.

A simple vista, una botella de dos polímeros como el PET (polietilen tereftalato) y el PVC (cloruro de polivinilo) pueden parecer iguales, pero químicamente son muy diferentes.

Teniendo en cuenta estos inconvenientes y para facilitar la clasificación de los polímeros, la Society of Plastics Industry desarrolló una codificación que se pone en la mayoría de los productos del polímero (Rodriguez, Cohen, Ober y Archer, 2014) (figura 1). 
Figura 1. Codificación de polímeros para su reciclaje

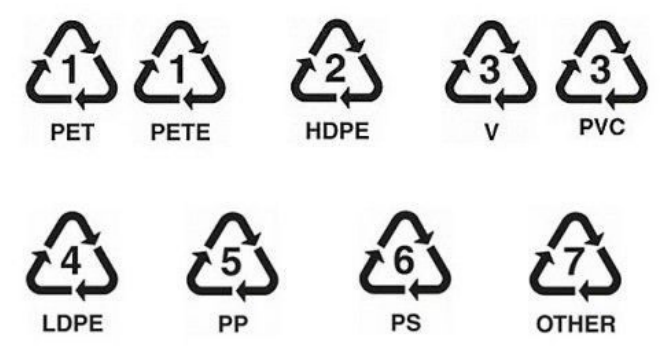

Fuente. Residuos Electrónicos, 2013.

Los símbolos clasifican los polímeros como:

- PET (polietilen tereftalato): uno de los polímeros más conocidos, que en general se encuentra en envases de bebidas y en algunos textiles (figura 2).

Figura 2. Fórmula química del PET

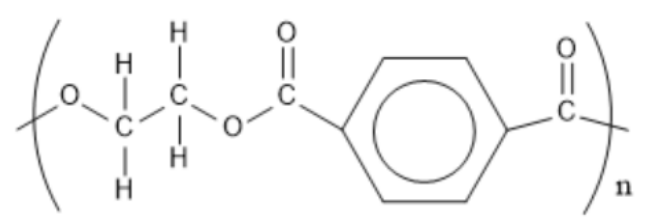

Fuente. Elaboración propia. 
- HDPE (polietileno de alta densidad): es un polímero más rígido y resistente que el PET, que se emplea también para elaborar envases plásticos desechables (figura 3).

Figura 3. Fórmula química del HDPE.

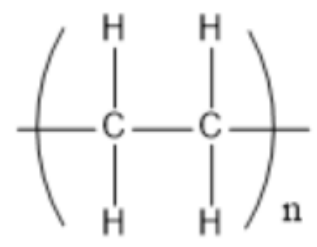

Fuente. Elaboración propia.

- PVC (policloruro de vinilo): un polímero bastante resistente y con muy buenas propiedades mecánicas, por lo que es común encontrarlo en productos para la construcción y edificación, como tejas y tuberías (figura 4).

Figura 4. Fórmula química del PVC

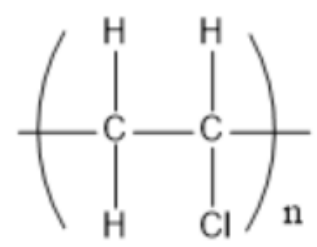

Fuente. Elaboración propia. 
- LDPE (polietileno de baja densidad): es un polímero más flexible que el HDPE; aunque químicamente son iguales, presenta diferencias en la polimerización que produce diferentes propiedades mecánicas y buena resistencia. Es común que sea utilizado para elaborar bolsas y envolturas (figura 5).

Figura 5. Fórmula química del polietileno de baja densidad

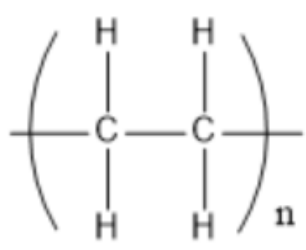

Fuente. Elaboración propia.

- PP(polipropileno): es un polímero con muy buenas propiedades mecánicas y resistencia a químicos. Se emplea para empaques de alimentos, algunos materiales para laboratorios y componentes especializados (figura 6).

Figura 6. Fórmula química del PP

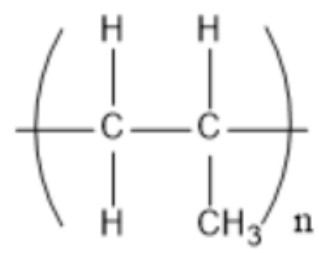

Fuente. Elaboración propia. 
- PS (poliestireno): es un polímero con bastantes aplicaciones industriales. Hay distintos tipos de este polímero y con aplicaciones comerciales muy diversas.

En Colombia, el nombre icopor (Jhonstevenmorenog, s. f.) es uno de los nombres que se le dio al poliestireno expandido por su fabricante, Industria Colombiana de Porosos (figura 7).

Figura 7. Fórmula química del PS

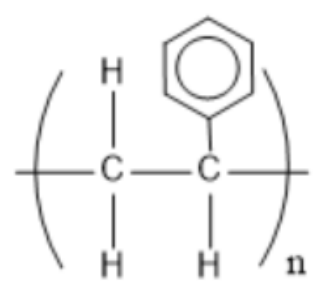

Fuente. Elaboración propia.

- Otros polímeros (como el PC policarbonato): este grupo incluye los otros tipos de polímeros, polímeros de procedencia biológica -biopolímeros - o aquellos que son una mezcla de varios plásticos — copolímeros-. Sus propiedades son muy diversas y van a depender de los monómeros que están conformados y de las proporciones de estos (figura 8).

Figura 8. Fórmula química del policarbonato

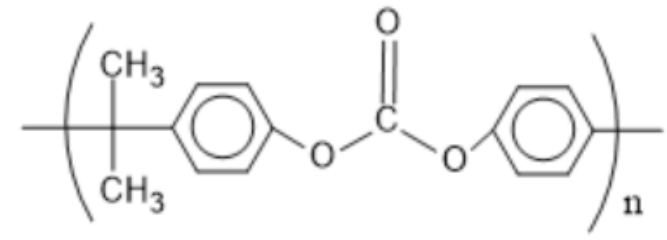

Fuente. Elaboración propia. 
El sistema de numeración no implica que todos los polímeros sean reciclados. Los polímeros como el PET y el HDPE son reciclados en la mayoría de los países realizando procesos de separación en general manual o apoyados en instrumentos que emplean la tecnología de espectrometría infrarroja, lo que hace que este proceso de separación de los polímeros sea bastante costoso y el reciclaje de los polímeros una labor poco o nada rentable (Al-Salem, Lettieri y Baeyens, 2009).

\subsection{Espectroscopía infrarroja y análisis de espectros}

La espectroscopía infrarroja es una de las técnicas de espectroscopía molecular, que, junto con la espectrofotometría UV-vis, permiten obtener información estructural de la materia.

En esta técnica, un haz de luz infrarroja incide sobre la materia y provoca vibraciones de los átomos de la molécula. Las vibraciones son específicas a determinadas frecuencias de los enlaces químicos, que corresponden a niveles de energía de la molécula, y van a depender de la forma de la superficie de energía potencial de la molécula, la geometría molecular, las masas atómicas $\mathrm{y}$, posiblemente, el acoplamiento vibracional.

La cantidad de luz absorbida es registrada continuamente, rango de longitudes de onda de interés, por lo general, 4000-400 $\mathrm{cm}^{-1}$ (Stuart, 2004) (figura 9). 
Figura 9. Diagrama de funcionamiento de un espectrómetro infrarrojo

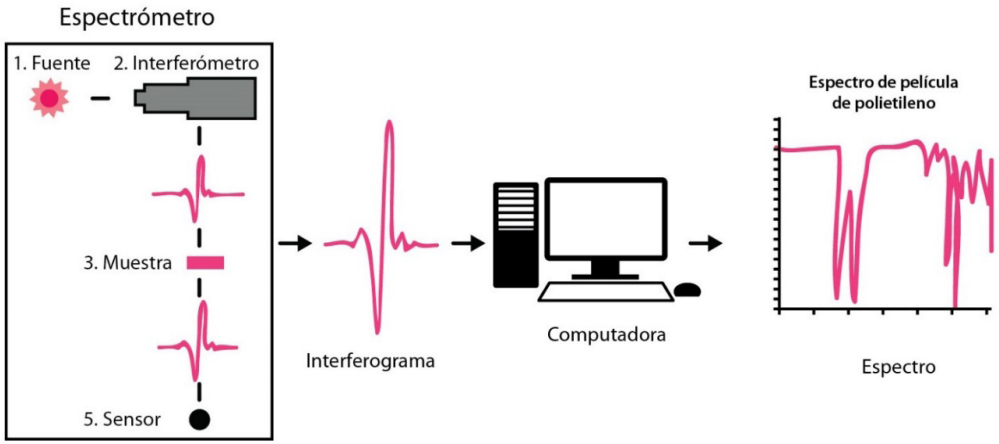

Fuente. Noria, 2017.

Dado que las bandas vibracionales son características para muchos grupos funcionales, se emplea los espectros como conjunto para identificar a las moléculas.

Los enlaces entre los átomos no son rígidos, son más bien como resortes. La figura representa algunos de los movimientos que se dan en los enlaces entre átomos (figura 10).

Figura 10. Algunas vibraciones típicas de los átomos

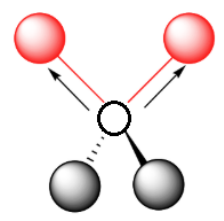

Estiramientos (simétricos y antisimétricos)

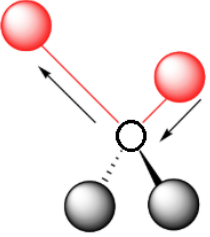

s)

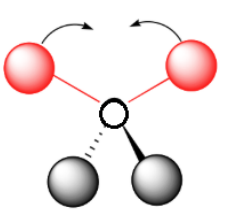

Flexiones (tijereteo o balanceo)

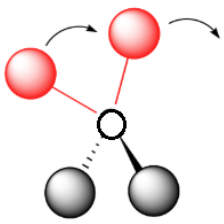

Fuente. Modificada a partir de Barraza-Garza, De la Rosa, Martínez-Martínez, CastilloMichel, Cotte y Alvarez-Parrilla, 2013.

Todas las vibraciones entre átomos se van a representar como bandas en un espectro. Ya es común encontrar tablas detalladas en las que se analizan los tipos de bandas de absorción, la región en la que ocurren y 
relacionarlas con grupos funcionales específicos, lo que permite realizar una elucidación estructural de las moléculas (Barraza-Garza et al., 2013).

El siguiente es el espectro típico del acetato de etilo. Se resaltan las bandas de absorción y se relacionan con algunos enlaces presentes en las moléculas (figura 11).

Figura 11. Espectro del acetato de etilo

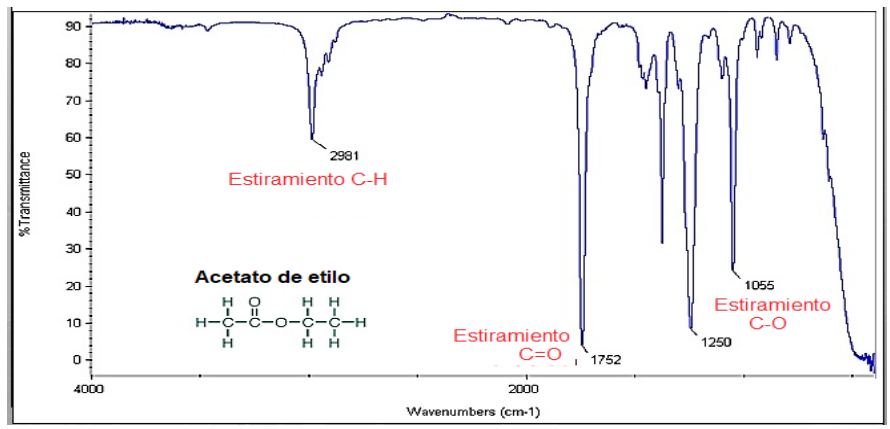

Fuente. Modificada a partir de Orgchemboulder.com, s. f.

\subsection{Funcionamiento de un espectrómetro infrarrojo}

Los espectrómetros infrarrojos basan su funcionamiento en el interferómetro de Michelson. En ellos, la radiación de la fuente pasa a través de un beam splitter que divide la radiación dirigiendo la mitad a un espejo fijo y la otra a un espejo móvil.

Los rayos se recombinan en el beam splitter de manera constructiva o destructiva dependiendo de la posición del espejo (figura 12). 
Figura 12. Partes de un espectrómetro infrarrojo
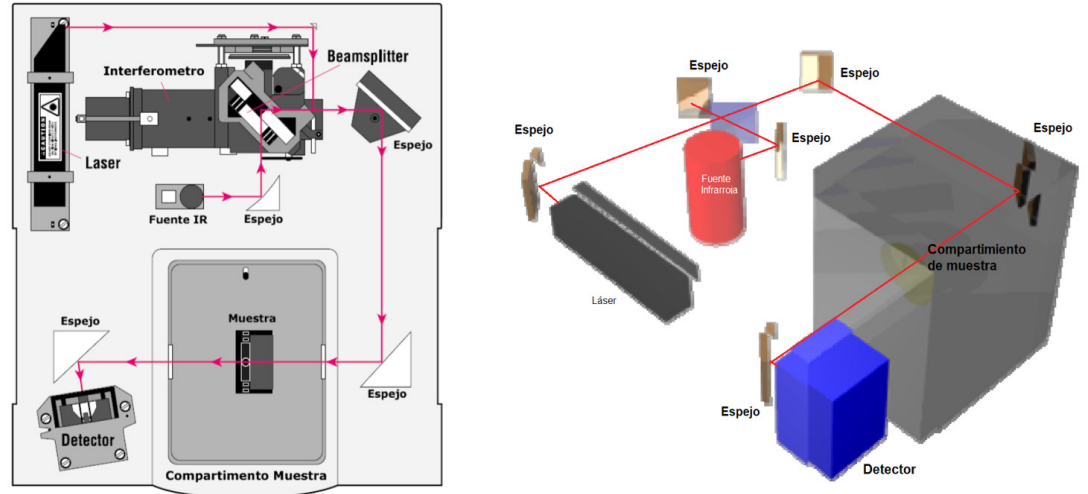

Fuente. Modificada a partir de Uhv.es, s. f.

El resultado es el interferograma —una representación de intensidad en función del tiempo- que se convierte con la transformada de Fourier en un diagrama que representa la intensidad en función de la frecuencia.

Es una de las técnicas analíticas más versátiles y de mayores aplicaciones, ya que permite realizar mediciones en cualquier tipo de muestra sin importar si es un líquido, sólido o gas, por lo que se han desarrollado accesorios especializados para disponer la muestra.

El más común y utilizado es el ATR —reflexión total atenuada—. En ese accesorio, el haz de infrarrojo es reflejado internamente por medio de espejos e incide sobre un cristal denso ópticamente con un alto índice de refracción en determinado ángulo. El cristal está en contacto con la muestra, de manera que el rayo incide sobre una distancia leve de la muestra (Figura 13). 
Figura 13. Diagrama de funcionamiento del accesorio ATR y accesorio ATR Specac
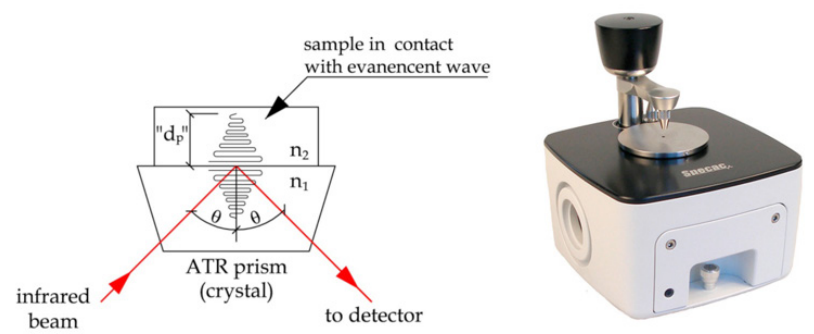

Fuente. Modificada a partir de Urbaniak-Domagala (2012) y de Specac.com, s. f.

Una de las grandes ventajas que ofrece el uso del ATR es que permite la lectura de muestras con una preparación mínima o sin preparación de la muestra.

La muestra se pone directamente sobre el disco cubriendo la superficie del cristal, lo que la hace ideal para la lectura de muestras líquidas o sólidas.

\section{Metodología}

\subsection{Instrumento empleado para los análisis}

El espectrómetro de infrarrojo es el IR-Tracer100 de Shimadzu con un ATR Specac que hace parte de los instrumentos de análisis químico del laboratorio de absorción atómica y cromatografía de la Universidad EAN con el software de adquisición de datos LabSolutions IR.

\subsection{Método de adquisición de datos}

Los espectros se toman en modo \% Transmitancia, con apodización Happ-Genzel en un rango entre $340-4700 \mathrm{~cm}^{-1}$ con una resolución de $2 \mathrm{~cm}^{-1}$. 
Para cada muestra se toman 45 scans (figura 14).

Figura 14. Espectrómetro infrarrojo del Laboratorio de Cromatografía y Absorción Atómica de la Universidad EAN

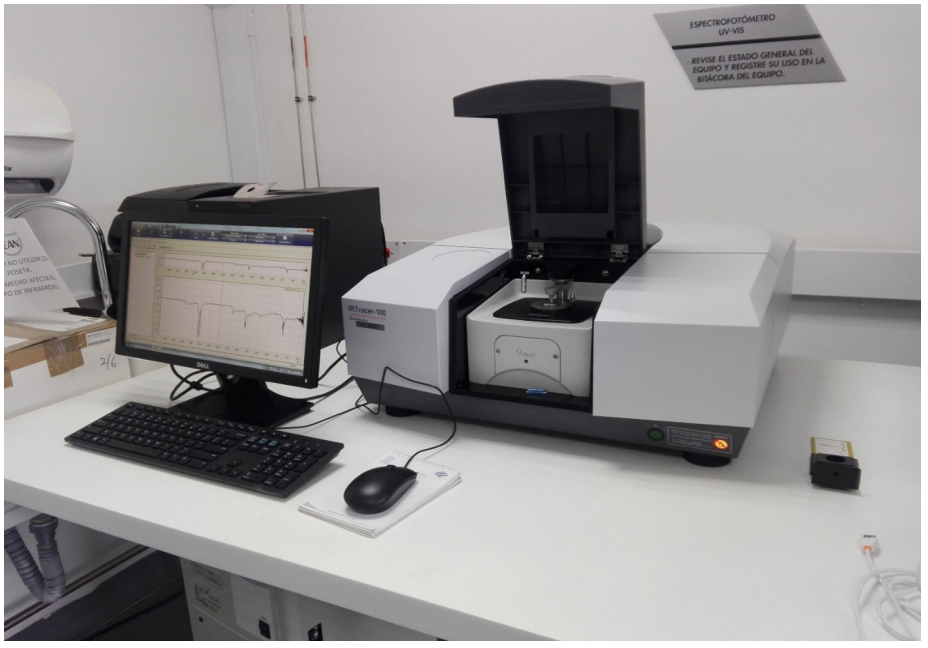

Fuente. Elaboración propia.

El software de adquisición de datos es el software LabSolutions IR de Shimadzu (figura 16).

Figura 15. Software LabSolutions IR del espectrómetro infrarrojo IR-Tracer100 de Shimadzu

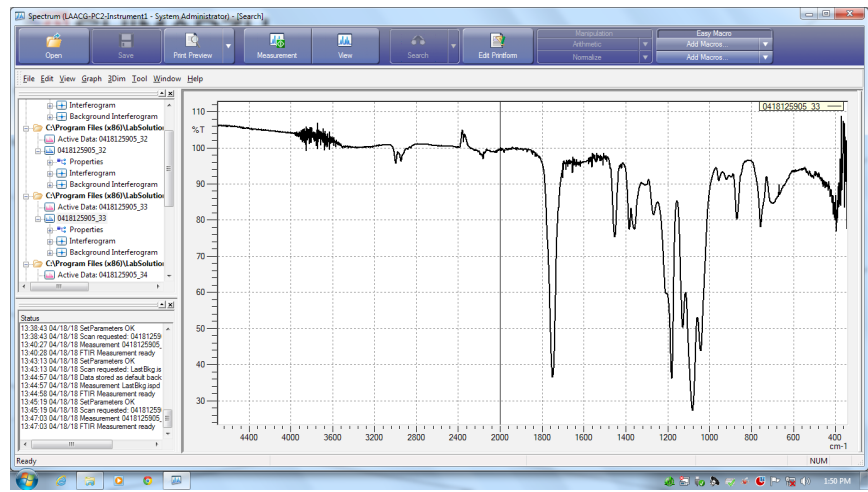

Fuente. Elaboración propia. 


\subsection{Toma de las muestras}

Se tomaron muestras de distintos polímeros al azar, obtenidos de materiales de uso cotidiano: bolsas plásticas, envases de bebidas, recipientes para alimentos, entre otros.

En todos los casos, se identificó el polímero de acuerdo con la clasificación para reciclaje y se cortó una lámina fina que se puso sobre el cristal del accesorio ATR.

Figura 16. Muestras de polímeros analizadas

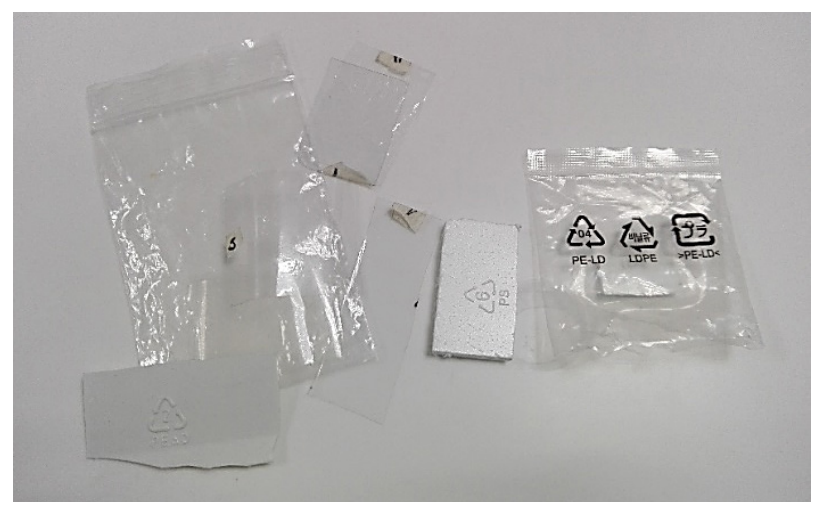

Fuente. Elaboración propia.

\section{Discusión de los resultados}

Los siguientes son los espectros resultados de cada uno de los polímeros y un breve análisis de las bandas de absorción.

\subsection{Espectro resultado de una muestra de PET (grupo 1)}

En este espectro, se observa una banda intensa correspondiente a la tensión del enlace $\mathrm{C}=\mathrm{O}$ en 1700 y movimientos de tensión entre 1000$1100 \mathrm{~cm}^{-1}$ por tensiones de los enlaces del anillo aromático. Las bandas de 
tensión de enlaces C-H a 2800-2900 $\mathrm{cm}^{-1}$ son débiles, aunque se alcanzan a percibir (figura 17).

Figura 17. Análisis del espectro de PET

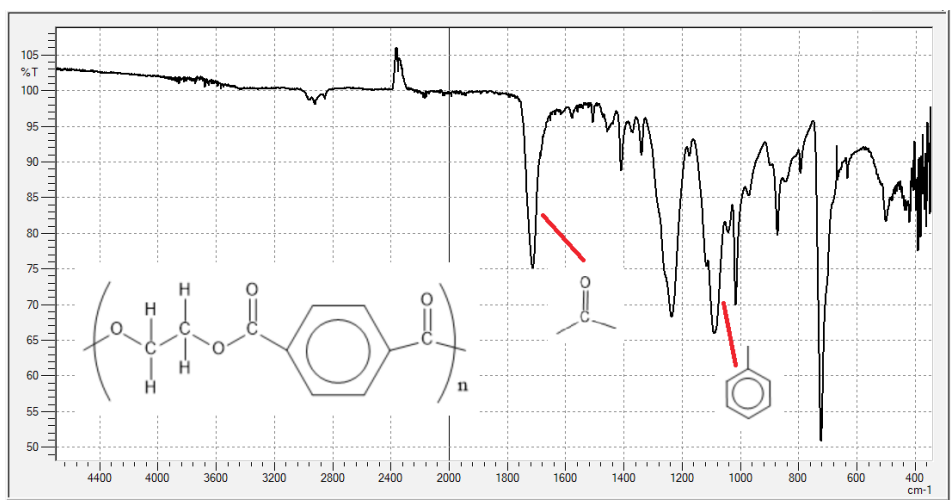

Fuente. Elaboración propia.

\section{Espectro resultado de una muestra de HDPE (grupo 2)}

En este espectro, se observan claramente tres grupos de bandas correspondientes a movimientos de tensión de los enlaces C-H a 2850 $\mathrm{cm}^{-1}$, tensión C-C a $1500 \mathrm{~cm}^{-1} \mathrm{y}$ a un movimiento de flexión de $-\mathrm{CH}_{2}$ en $700 \mathrm{~cm}^{-1}$.

Dado que químicamente el HDPE es igual al LDPE, las bandas de absorción son las mismas (figura 18). 
Figura 18. Análisis del espectro de polietileno de alta densidad

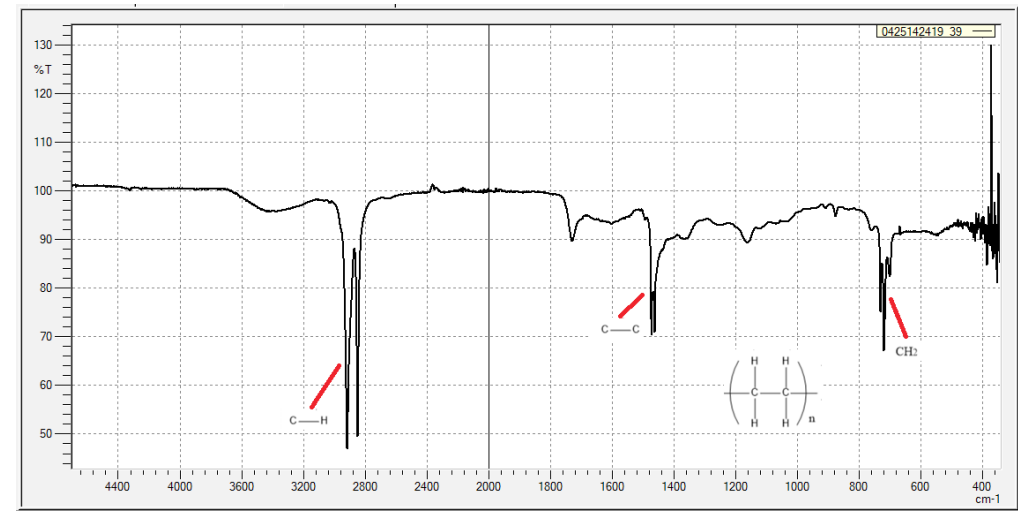

Fuente. Elaboración propia.

\subsection{Espectro resultado de una muestra de PVC (grupo 3)}

La posición de las bandas de absorción es similar a la del polietileno, sin embargo, se observa un efecto por la presencia de cloro, bandas fuertes entre 1000-1100 $\mathrm{cm}^{-1}$. Se advierte además una banda de tensión de los enlaces C-H a 2850-2900 cm-1, otra de tensión C-C a $1250 \mathrm{~cm}^{-1}$ y a un movimiento de flexión de $-\mathrm{CH}_{2}$ en $720-730 \mathrm{~cm}^{-1}$ (figura 19).

Figura 19. Análisis del espectro de PVC

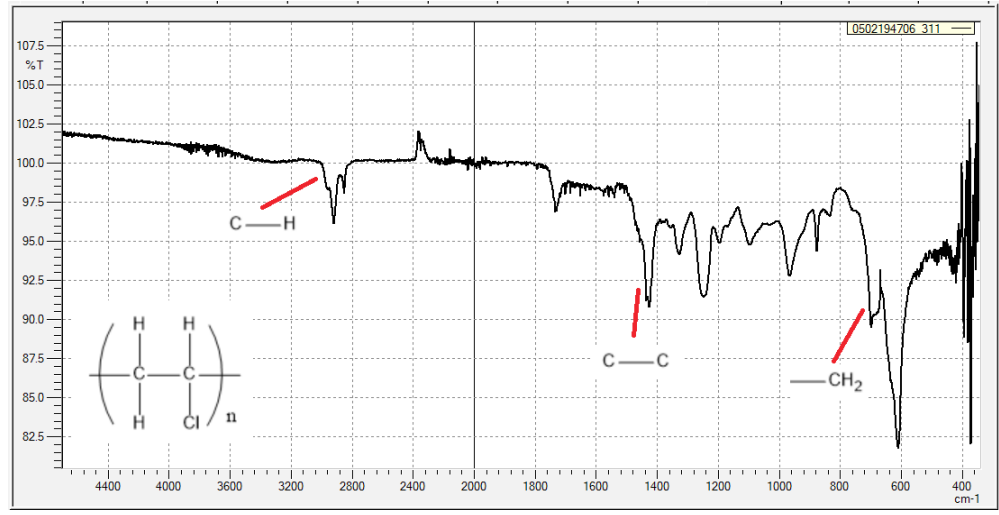

Fuente. Elaboración propia. 


\subsection{Espectro resultado de una muestra de LDPE}

\section{(grupo 4)}

En este espectro, se observan claramente tres grupos de bandas correspondientes a movimientos de tensión de los enlaces C-H a 2900 $\mathrm{cm}^{-1}$ y C-C $1450 \mathrm{~cm}^{-1}$ y a un movimiento de flexión de $-\mathrm{CH}_{2}$ en $750 \mathrm{~cm}^{-1}$ (figura 20).

Figura 20. Análisis del espectro de LDPE

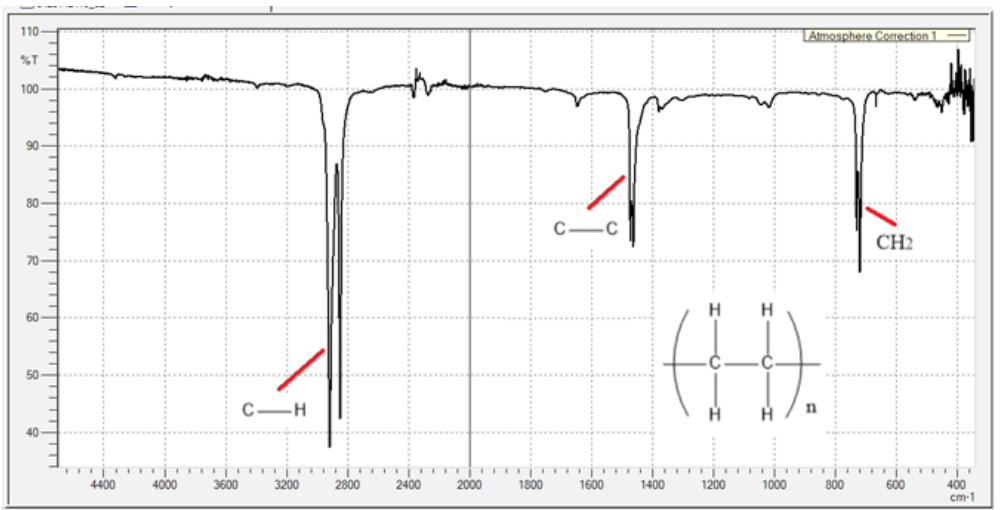

Fuente. Elaboración propia.

\subsection{Espectro resultado de una muestra de PP (grupo 5)}

En este espectro, se observan claramente tres grupos de bandas correspondientes a movimientos de tensión de los enlaces C-H a 2900 $\mathrm{cm}^{-1} \mathrm{y}$ movimientos de tensión C-C en $1350-1450 \mathrm{~cm}^{-1} \mathrm{y}$ a movimientos de flexión de $-\mathrm{CH}_{3}$ entre $1200-1000 \mathrm{~cm}^{-1}$ (figura 21). 
Figura 21. Análisis del espectro de PP

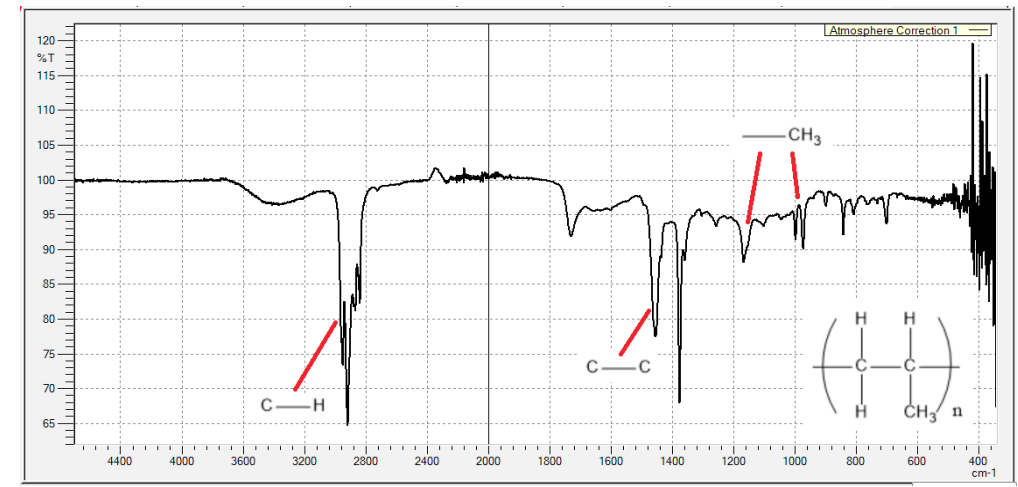

Fuente. Elaboración propia.

\subsection{Espectro resultado de una muestra de PS (grupo 6)}

En este espectro, se observan claramente tres grupos de bandas correspondientes a los múltiples movimientos de tensión de los enlaces C-H a 2800-3200 $\mathrm{cm}^{-1}$, C-C $1400-1600 \mathrm{~cm}^{-1}$ del anillo aromático y a movimiento de flexión de $-\mathrm{CH}_{2} \mathrm{y}$ tensiones del anillo aromático entre $700-800 \mathrm{~cm}^{-1}$ (figura 22).

Figura 22. Análisis del espectro de PS

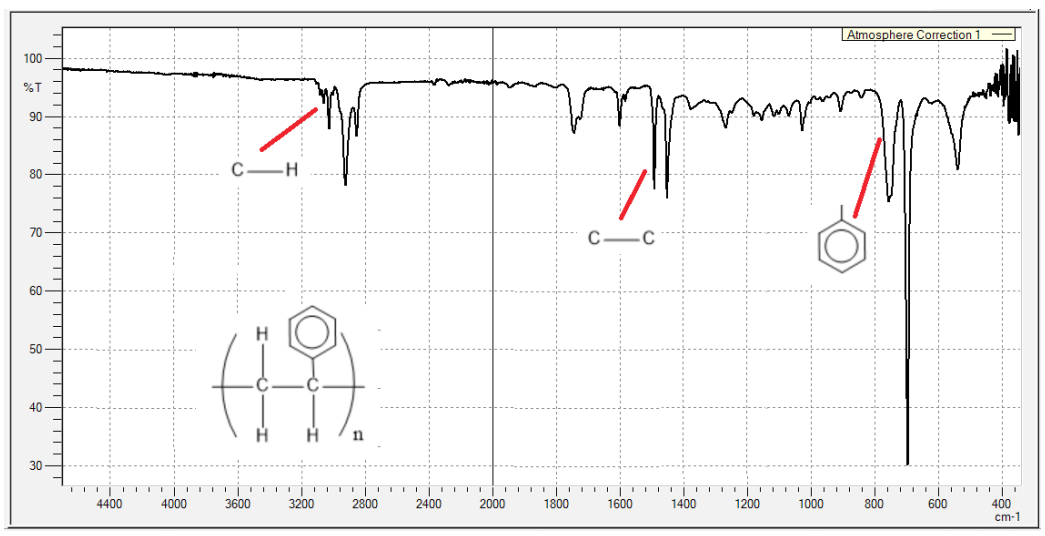

Fuente. Elaboración propia. 


\subsection{Espectro resultado de una muestra de PC (grupo 7)}

En este espectro, se observan bandas correspondientes a movimientos de tensión de los enlaces $\mathrm{C}-\mathrm{H}$ a $2800-3000 \mathrm{~cm}^{-1}$, un movimiento de tensión que es bastante notorio y que es bastante específico es el de $\mathrm{C}=\mathrm{O}$ alrededor de $1750 \mathrm{~cm}^{-1}$, movimientos de tensión de enlaces C-C 1400$1600 \mathrm{~cm}^{-1} \mathrm{y}$ a un movimiento de flexión y de tensión de $-\mathrm{CH}_{2}$ aromático y $-\mathrm{CH}_{3}$ entre $500-1300 \mathrm{~cm}^{-1}$ (figura 23).

Figura 23. Análisis del espectro de PC

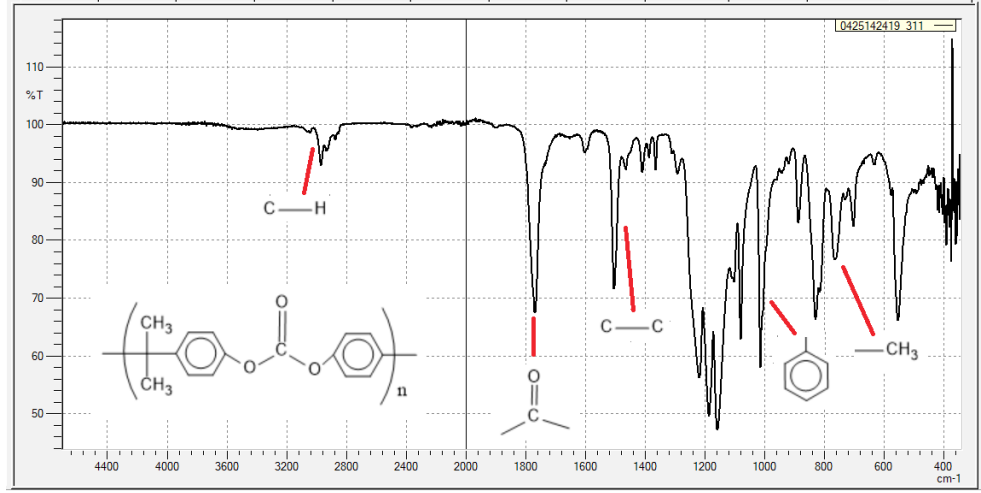

Fuente. Elaboración propia.

De acuerdo con los espectros obtenidos, se observa que hay bandas características para cada uno de los polímeros analizados.

Los resultados que se obtienen son bastante buenos, teniendo en cuenta que no se trabajó con materiales puros, sino con materiales disponibles comercialmente, que siempre están mezclados con pigmentos o con otras sustancias en pequeñas cantidades que pueden actuar como interferentes o ruido en el espectro.

La figura 24 reúne los espectros obtenidos. Se evidencian bandas de absorción comunes, típicamente las que ocurren en la región de 2800$2900 \mathrm{~cm}^{-1}$, un pico intenso por la tensión de $\mathrm{C}=\mathrm{O}$ alrededor de 1750 
$\mathrm{cm}^{-1}$, movimientos de tensión de enlaces C-C $1400-1600 \mathrm{~cm}^{-1}$ y a un movimiento de flexión y de tensión de $-\mathrm{CH}_{2}$ aromático y $-\mathrm{CH}_{3}$ entre $500-1300 \mathrm{~cm}^{-1}$.

Figura 24. Comparación de los espectros obtenidos

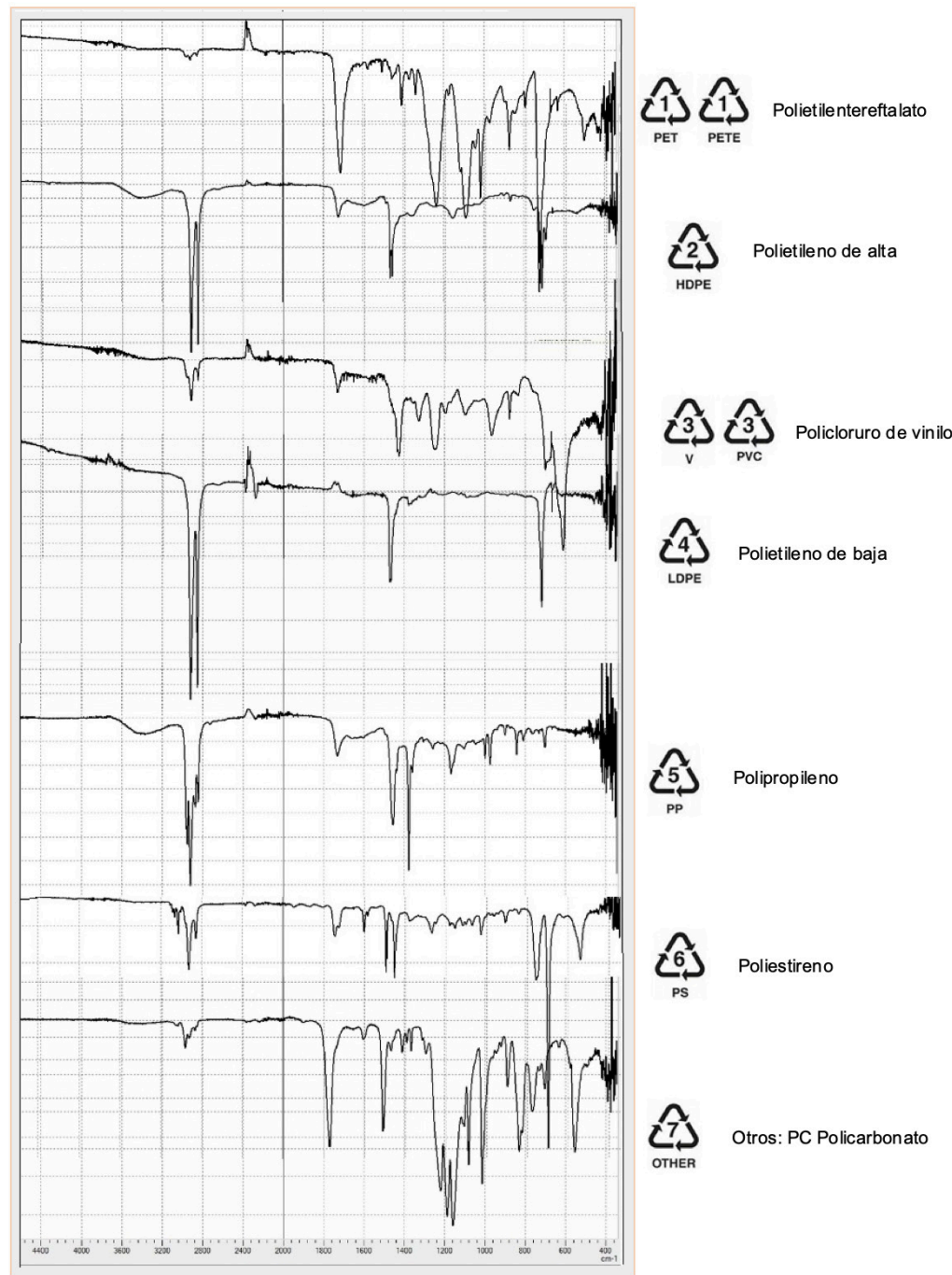

Fuente. Elaboración propia. 


\section{Conclusiones}

e los resultados obtenidos se concluye que la espectrometría infrarroja con el accesorio ATR ofrece una alternativa para identificar rápidamente un polímero y es una herramienta bastante útil para elucidar información sobre la estructura química de este.

Las muestras de polímeros analizadas se tomaron teniendo en cuenta la clasificación de la Society of Plastics Industry, con muestras aleatorias de plásticos empleados en bolsas, botellas y empaques, a las que no se les realizó ningún tratamiento y que se midieron directamente.

La información obtenida con el espectrómetro infrarrojo es bastante útil para entender las diferencias en composición química de los polímeros, y muestra que algunos de los polímeros estudiados se componen estructuralmente de las mismas subunidades y presentan tensiones en regiones comunes del espectro: Tensiones C-C en la región de 1400-1600 cm $\mathrm{cm}^{-1}$, tensiones C-H en la región de 2800-3200 $\mathrm{cm}^{-1}$, tensión de $-\mathrm{CH}_{2} \mathrm{y}-\mathrm{CH}_{3}$ alifático o aromático entre $500-1300 \mathrm{~cm}^{-1}$ (en polímeros como el LDPE, HDPE o PP, entre otros), o aquellos que tienen el grupo carbonilo $\mathrm{C}=\mathrm{O}$ en su estructura y una tensión de enlace fuerte en 1700 $\mathrm{cm}^{-1}$ (como en el PET y el PC).

Los espectros obtenidos se compararon con los que están disponibles en la librería incorporada del instrumento y siempre hubo coincidencia en los resultados obtenidos, lo cual demostró que la técnica es muy robusta y que puede aplicarse con bastante confianza para realizar la identificación rápida de polímeros.

Dado que la industria de reciclaje de polímeros está muy poco desarrollada, vale la pena invertir esfuerzos e investigaciones en torno a este tema, ya que estos materiales se convirtieron en uno de los principales 
problemas de contaminación por la cantidad de residuos de este tipo que son desechados (Rodriguez, Cohen, Ober y Archer, 2014).

Por el momento, como consumidores de polímeros, se recomienda reusarlos siempre que se pueda y extender su vida útil el mayor tiempo posible.

Los resultados obtenidos dejan ver la utilidad que se puede dar a este equipo para continuar realizando investigaciones relacionadas con los polímeros, como efectuar seguimiento a las reacciones de polimerización, caracterizaciones de las estructuras del polímero, y examinar las superficies de los polímeros o los procesos de degradación.

Con modelos matemáticos más avanzados y realizando análisis multivariados, es posible, incluso, llegar a proponer modelos para la cuantificación de polímeros en mezclas. 


\section{Referencias}

Al-Salem, S. M., Lettieri, P. y Baeyens, J. (2009). Recycling and recovery routes of plastic solid waste (PSW): A review. Waste Management, 29(10), 2625-2643. https://doi.org/10.1016/j.wasman.2009.06.004

Barraza-Garza, G., de la Rosa, L. A., Martínez-Martínez, A., Castillo-Michel, H., Cotte, M. y Alvarez-Parrilla, E. (2013). La microespectroscopía de infrarrojo con transformada de Fourier (FTIRM) en el estudio de sistemas biológicos. Revista Latinoamericana de Química, 41(3), 125-148.

Recuperado de https://bit.ly/2w4cjSq

Eisenreich, N. y Rohe, T. (2006). Infrared spectroscopy in analysis of plastics recycling. En encyclopedia of analytical chemistry: Applications, theory and instrumentation. Hoboken, NJ: John Wiley \& Sons. https://doi. org/10.1002/9780470027318.a2011

Escuela Colombiana de Ingeniería (2008). Identificación de plásticos. Manuscrito no publicado, Facultad Ingeniería Industrial, Escuela Colombiana de Ingeniería, Colombia.

Flory, P. J. (1953). Principles of polymer chemistry. Ithaca: Cornell University Press.

Ignatyev, I. A., Thielemans, W. y Vander Beke, B. (2014). Recycling of polymers: A review. ChemSusChem, 7(6), 1579-1593. https://doi. org/10.1002/cssc. 201300898

Jhonstevenmorenog (s. f.). Nombre cientifico del icopor. Recuperado de https://bit.ly/2VumEBc

Orgchemboulder.com (s. f.). IR Spectroscopy Tutorial: Esters. Recuperado de https://bit.ly/2HpcXyY

Noria (2017, noviembre 10). Beneficios del análisis FTIR. Recuperado de https://bit.ly/2Q40owY

Residuos Electrónicos (2013, mayo 20). La simbología del reciclaje. Recuperado de https://bit.ly/2EpBvHN 
Rodriguez, F., Cohen, C., Ober, C. K. y Archer, L. (2014). Principles of polymer systems. Boca Raton: CRC Press.

Specac.com (s. f.). Quest ATR FTIR. Recuperado de https://bit.ly/30nJpKX

Stuart, B. H. (2004). Infrared spectroscopy: Fundamentals and applications. Hoboken, NJ: John Wiley \& Sons.

Uhv.es (s. f.). Espectrometría infrarroja. Recuperado de https://bit. ly/2Q6PGpz

Urbaniak-Domagala, W. (2012). The use of the spectrometric technique FTIR-ATR to examine the polymers surface. En W. Urbaniak-Domagala (ed.), Advanced aspects of spectroscopy (pp. 85-104). Rijeka, Croacia: InTech. http://dx.doi.org/10.5772/2757 